\title{
A THREE DIMENSIONAL LOCALISER FOR AUTONOMOUS ROBOT VEHICLES1
}

\author{
Lindsay Kleeman \\ Intelligent Robotics Research Centre \\ Department of Electrical and Computer Systems Engineering \\ Monash University, Clayton VIC 3168
}

Australia

\begin{abstract}
A novel design of a three dimensional localiser intended for autonomous robot vehicles is presented. A prototype is implemented in air using ultrasonic beacons at known positions, and can be adapted to underwater environments where it has important applications, such as deep sea maintenance, data collection and reconnaissance tasks. The paper presents the hardware design, algorithms for position and orientation determination (six degrees of freedom), and performance results of a laboratory prototype. Two approaches are discussed for position and orientation determination (i) fast single measurement set techniques and (ii) computationally slower Kalman filter based techniques. The Kalman filter approach allows the incorporation of robot motion information, more accurate beacon modelling and the capability of processing data from more than four beacons, the minimum number required for localisation.
\end{abstract}

1 This work was supported by a Monash University Special research grant. 


\section{Keywords}

Position estimation, 3-D localisation, Kalman filtering, ultrasonic navigation, orientation, underwater vehicles. 


\section{Introduction}

Untethered underwater robot vehicles present challenging problems to the robotics researcher over conventional wheeled vehicles operating on a plane surface due to the extra degrees of freedom in movement and lack of rigid control 1,2,3,4,5. Localisation in difficult environments, such as in dark or muddied waters with temperature gradients and varying currents, is an important research issue, especially in light of the fact that these conditions are present over most of the Earth. The exploration and exploitation of undersea regions has immense potential which can be achieved through autonomous undersea vehicles with the ability to determine their position accurately in three dimensions.

The three dimensional localiser described in this paper can measure the full six degrees of freedom of position and orientation. Untethered physical autonomy is a feature of this localiser since neither physical connections nor communication links to the environment are necessary. Applications to autonomous airborne and underwater robot vehicles are feasible, even in poor visibility conditions. The localisation system relies on artificial beacons and hence is independent of environmental features, such as a sea-bed or underwater structures and can operate in open space where no identifiable features exist. Other approaches to localisation can exploit environmental features $1,3,5$. The paper describes algorithms for robustly determining position and orientation so that sonar reflections and obscured beacons have little effect on accuracy.

A localiser based on three artificial beacons has been reported that produces two dimensional position from the intersection of hyperboloids and relies on under-water pressure sensors for the 
depth $^{4}$. Other localisation systems exist for underwater navigation, such as the SHARPS system ${ }^{2}$ which reports the vehicle $2 \mathrm{D}$ position, heading and roll using a beacon system. The work presented here uses a fourth beacon for determining depth, and Kalman filtering is employed for optimal position and orientation estimates with the rejection of spurious data. Orientation is available from the system presented here due to the receiver array structure employed. The system presented here is novel in the sense that it produces robust estimates of all the six degrees of freedom of position and orientation with the one sensor.

The work described here is an extension of a two dimensional localiser6,7. This paper significantly improves on previous work on a three dimensional position determination algorithm 8 and orientation determination ${ }^{9}$. The organisation of the paper is as follows: In section 2 the structure of the localiser is introduced and section 3 outlines the hardware design of an airborne prototype. In Section 4 two position determination algorithms are presented, one derived from geometrical constraints and the other taking into account models of robot motion and beacon behaviour using an Iterated Extended Kalman Filter. In section 5, orientation algorithms are derived, and results of experiments on the prototype are summarised in section 6 . Finally future work and conclusions are presented in section 7 .

\section{Overview of the 3-D Localiser}

The localiser requires at least four fixed beacons in known noncoplanar locations. For example, three beacons could be placed on the surface of water and the fourth suspended underwater. In the prototype described in this paper beacons are placed at the coordinate system origin and on each of the axes as shown in figure 1 . The 
beacons fire ultrasonic pulses that are received by the receiver array onboard the robot.

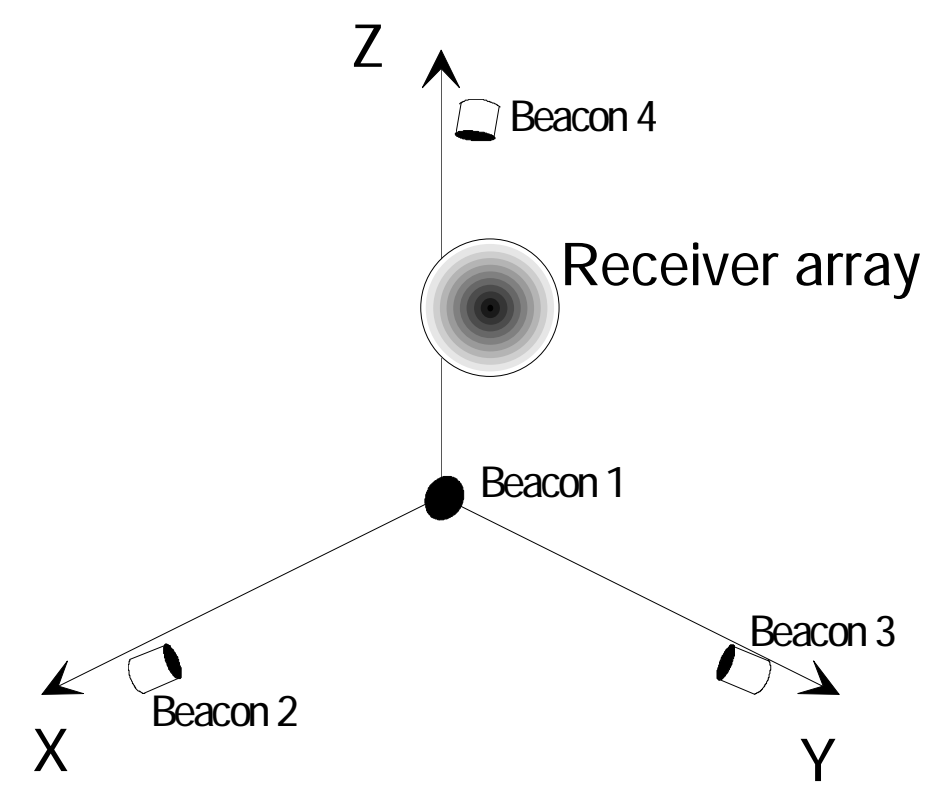

Figure 1 - Overview of Localiser (not to scale).

The beacons fire in a regular sequence with fixed inter-firing period as illustrated in figure 2. Note that beacon 1 emits a double pulse for initial identification by the receiver. Once beacon 1 is identified, the double pulse can be ignored, since the time of arrival uniquely determines the beacon number thereafter. The beacon design is similar in concept to the 2-D localisation system and is described in more detail elsewhere 6 . The beacons are required to be synchronised to each other, a common feature of many navigation systems. The synchronisation can be achieved via wiring interconnections as is done in the air prototype or via more sophisticated local time keeping and message passing approaches as in the Loran- $\mathrm{C}^{10}$ navigation system. 
Beacon 1

Beacon 2

Beacon 3

Beacon 4

Figure 2 - Beacon Firing Sequence (not to scale).

\section{Receiver Hardware Design}

The receiver array is spherical in shape and consists of an array of 32 ultrasonic receivers positioned on the faces of a truncated icosahedron as shown in figure 3. This arrangement orients all receivers so that their angular sensitivity adequately covers a full solid angle. There are two types of receivers - pentagonal receivers (connect to 5 adjacent receivers) and hexagonal receivers. The directions of a pair of adjacent receivers differ by 37.3 or 41.8 degrees, depending on whether the pair consists of two hexagonal receivers or one hexagonal and one pentagonal receiver. The worse case of 41.8 degrees corresponds to an angular sensitivity of $\quad-15 \mathrm{~dB}$ with respect to straight ahead.

The organisation of the 32 receivers for the interfacing to an 8 bit microprocessor is done by "colouring" the receivers with four colours so that no two adjacent receivers share the same colour, as shown in Figure 4. The colouring allows receivers to be interfaced in four groups where at most one receiver within a group is active for any arrival pulse direction. Interference is thus avoided within a group interface where signals are added before being rectified for envelope extraction. 
The microprocessor interface circuit in figure 5 allows three modes of data interrogation: (i) the envelope of the sum of like coloured receiver signals, (ii) the envelope of the sum of all receiver signals (iii) the envelope of each receiver individually. The arrival time can then be logged accurately (within $40 \mu \mathrm{sec}$ ) and the closest receiver found. Due to the slow rise time of the received pulse envelope 0.5 msec), the closest receiver will have the maximum envelope amplitude just after a threshold has been exceeded by the sum of receiver signals. 
Please use photo original submitted with first draft.

Figure 3 Photo of Receiver Prototype Construction 


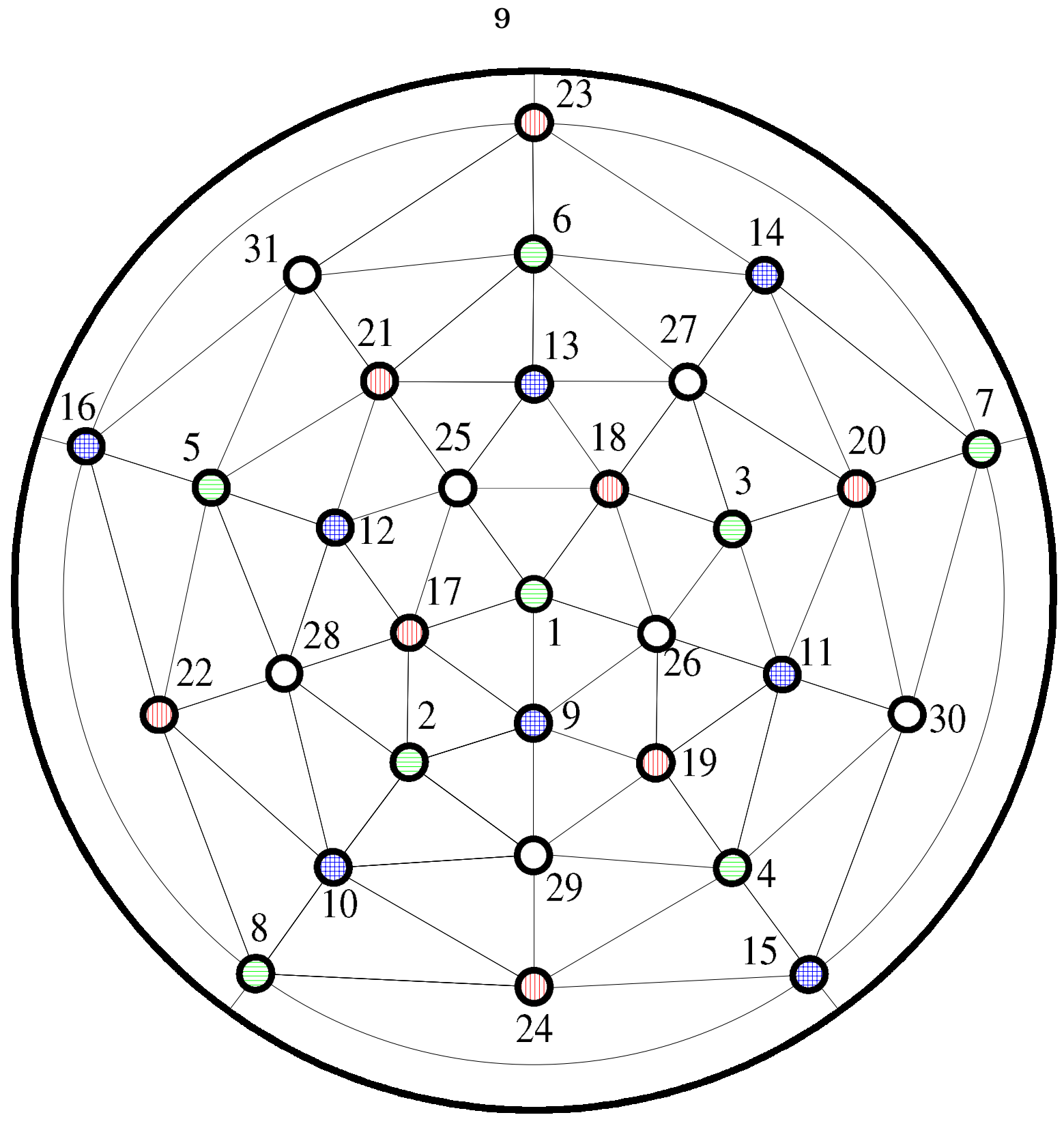

32

$\begin{array}{llll}\text { Oreen sensor } & \text { O Blue sensor } \\ & \text { Red sensor } & \text { O Black sensor }\end{array}$

Figure 4 Receiver array colouring. 

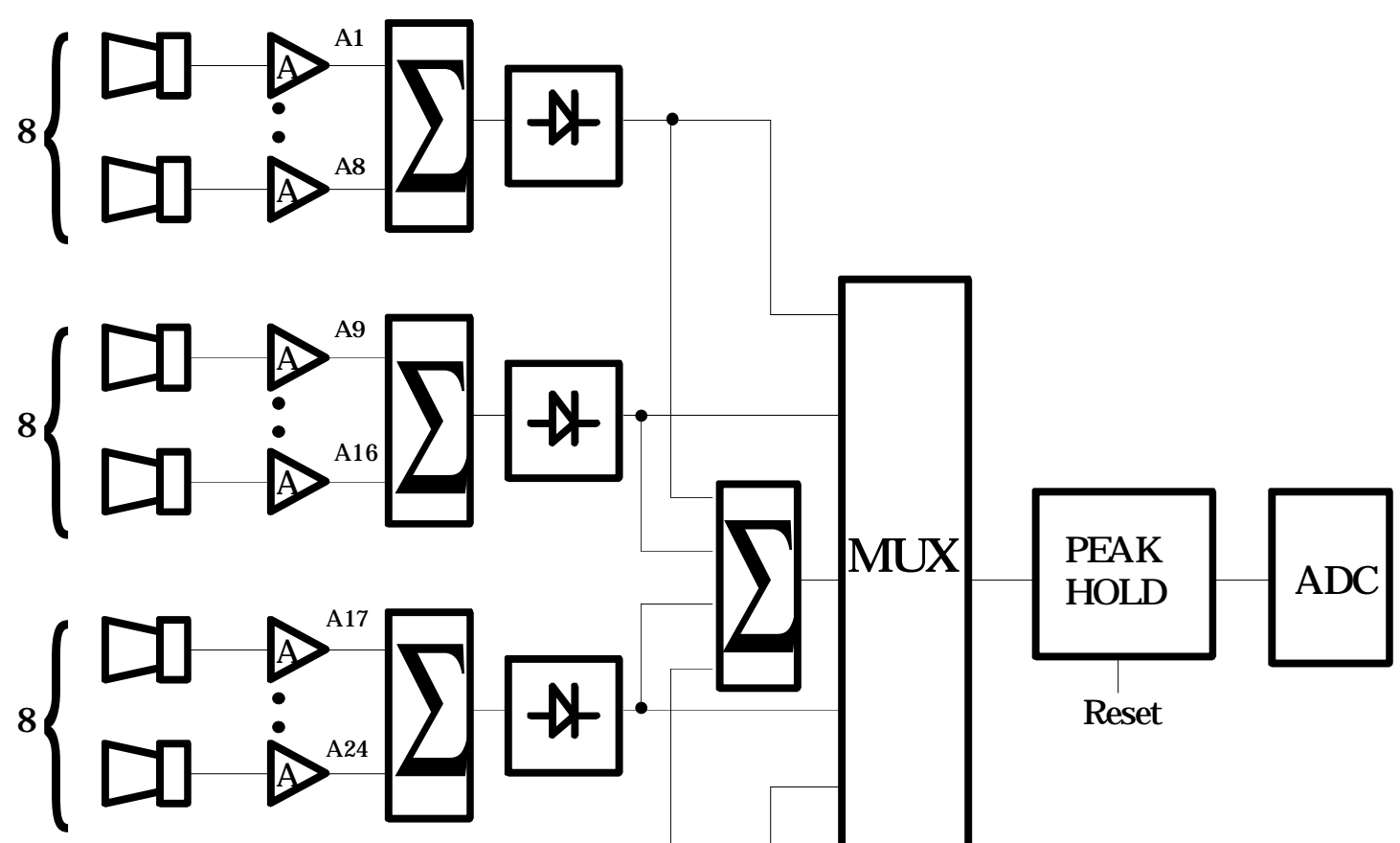

Reset
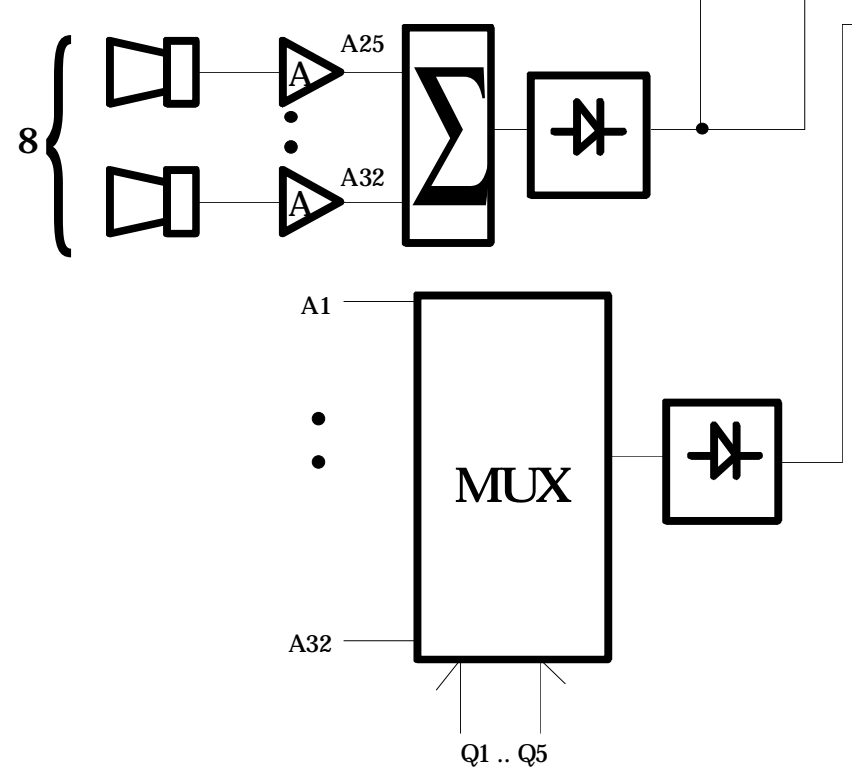

Q6.. Q8

Figure 5 Receiver Interface Circuitry.

The sequence of microprocessor interrogation begins by sampling the sum of all receivers in a tight loop until a threshold is exceeded. This time is recorded as the raw arrival time which is later compensated for the amplitude of the received pulse and the slow rise time. Each of the four coloured group sums is sampled to find the maximum colour, and then eight receivers are sampled to find the 
"maximum receiver" within this colour and hence overall. The maximum receiver provides an approximate estimate of the arrival direction of the pulse and is used to determine the orientation of the receiver. Thus, 12 receiver samples are necessary to find the maximum receiver as opposed to 32 that would be required with no receiver colour grouping.

\section{Position Determination}

Two techniques are described for 3D position determination. The first technique, called the geometric position algorithm, requires little computational time. The second is based on an Iterated Extended Kalman Filter and is superior in accuracy, flexibility and output rate, but requires a significant number of floating point operations per measurement.

\subsection{Geometric Position Algorithm}

An iterative algorithm is derived in another paper 8 based on the intersection of hyperboloids. In this paper, a more concise derivation of the same algorithm is presented based on the gradients of three scalar fields. The scalar fields consist of the difference in distances from a reference beacon to the other beacons. Differences in distances are considered since the absolute distances are unknown. To elaborate on this, consider the arrival time, $t_{i}$, corresponding to beacon $i(1 . .4)$

$$
t_{i}=(i-1) T+t_{0}+d_{i} / c
$$

where $T$ is the (known) beacon inter-firing period, $t_{O}$ is the (unknown) time when beacon 1 fires, $d_{i}$ is the distance to beacon $i$ and $c$ is the 
speed of sound (known). In order to eliminate the unknown $t_{0}$, differences in distances are employed.

Suppose the robot vehicle is at position $(x, y, z)$ and beacon $i$ at position $\left(x_{i}, y_{i}, x_{i}\right)$ with respect to an absolute coordinate frame. Let $d_{i j}$ be the difference in distance $d_{j}-d_{i}$, between the two distinct beacons $i$ and $j$.

$$
d_{i j}=\sqrt{\left(x-x_{j}\right)^{2}+\left(y-y_{j}\right)^{2}+\left(z-z_{j}\right)^{2}}-\sqrt{\left(x-x_{i}\right)^{2}+\left(y-y_{i}\right)^{2}+\left(z-z_{i}\right)^{2}}
$$

The difference in distance $d_{i j}$ can be considered as a scalar field. The aim of the position determination algorithm is to find the three dimensional position that has the required differences in distance to match the measurements $d_{12}, d_{13}$ and $d_{14}$ available from the localiser receiver. The approach taken is to start with an estimate of the position and repeatedly move by $\delta \boldsymbol{r}$ until errors in the differences of distance, $\delta d_{i j}$, are sufficiently small. The errors in differences of distance determine the next move via the gradient of the scalar field $d_{i j}$ as shown below.

The gradient of $d_{i j}$, denoted by $\nabla \mathbf{d}_{\mathrm{ij}}$, is obtained by differentiating equation (2) with respect to each coordinate to give the following simple expression

$$
\nabla \mathbf{d}_{\mathrm{ij}}=\mathbf{u}_{\mathrm{j}}-\mathbf{u}_{\mathrm{i}}
$$

where $\mathbf{u}_{\mathbf{i}}$ is the unit distance vector from beacon $i$ to the robot. The required incremental displacement vector, $\delta \boldsymbol{r}$, can be expressed in terms of a new set of three basis vectors, called a biorthogonal basis, where each basis vector is orthogonal to two of the gradient vectors 
and also self orthogonal. The coordinates, A, B and C, of $\delta \boldsymbol{r}$ with respect to the biorthogonal basis are defined by

$$
\delta \mathbf{r}=A\left(\nabla \mathbf{d}_{13} \times \nabla \mathbf{d}_{14}\right)+B\left(\nabla \mathbf{d}_{14} \times \nabla \mathbf{d}_{12}\right)+C\left(\nabla \mathbf{d}_{12} \times \nabla \mathbf{d}_{13}\right)
$$

where $x$ is the vector cross product. To a first order linear approximation, a small move $\delta \boldsymbol{r}$ will produce a change in $d_{i j}$ of $\delta d_{i j}$ if

$$
\delta \mathbf{r} \bullet \nabla \mathbf{d}_{\mathrm{ij}}=\delta d_{i j}
$$

where $\bullet$ is the vector dot product. Applying equation (5) to (4), allows solution for A, B and C as follows:

$$
\begin{aligned}
& A=\frac{\delta d_{12}}{\left(\nabla \mathbf{d}_{13} \times \nabla \mathbf{d}_{14}\right) \bullet \nabla \mathbf{d}_{12}} \\
& B=\frac{\delta d_{13}}{\left(\nabla \mathbf{d}_{14} \times \nabla \mathbf{d}_{12}\right) \bullet \nabla \mathbf{d}_{13}} \\
& C=\frac{\delta d_{14}}{\left(\nabla \mathbf{d}_{12} \times \nabla \mathbf{d}_{13}\right) \bullet \nabla \mathbf{d}_{14}}
\end{aligned}
$$

Applying the vector identities

$$
(\mathbf{x} \times \mathbf{y}) \bullet \mathbf{z}=(\mathbf{y} \times \mathbf{z}) \bullet \mathbf{x}=(\mathbf{z} \times \mathbf{x}) \bullet \mathbf{y}
$$

to equation (6) and substituting in equation (4) gives

$$
\delta \mathbf{r}=\frac{\delta d_{12}\left(\nabla \mathbf{d}_{13} \times \nabla \mathbf{d}_{14}\right)+\delta d_{13}\left(\nabla \mathbf{d}_{14} \times \nabla \mathbf{d}_{12}\right)+\delta d_{14}\left(\nabla \mathbf{d}_{12} \times \nabla \mathbf{d}_{13}\right)}{\left(\nabla \mathbf{d}_{13} \times \nabla \mathbf{d}_{14}\right) \bullet \nabla \mathbf{d}_{12}}
$$

The expression in equation (8) can be shown, not surprisingly, to give the same result if the reference beacon 1 is replaced by any other beacon and therefore the choice of beacon 1 as reference is arbitrary. 
Equations (1) and (3) combined with (8) define the iteration step for the solution of position. Equation (8) also allows the error in position to be estimated from errors in the difference in distances, $\delta d_{i j}$.

Each new position can be calculated in a few milliseconds on an $8028616 \mathrm{MHz}$ computer with only a 3 or 4 iterations required for convergence. The most significant component of the measurement delay is the inter-firing period which has been designed so that reverberating pulse amplitudes decay below the receiver threshold. Measurements are performed every 900 msec by the prototype.

\subsection{Iterated Extended Kalman Filter for Position Estimation}

Kalman filtering is a well established technique for estimating the state of a system in the presence of noise and the unfamiliar reader is referred to Jazwinski 11 for details. The Extended Kalman filter implementation of the localiser exploits previous estimates of position by using knowledge about the robot motion and beacon behaviour to smooth the data. The previous geometric position algorithm does not exploit this information since it processes each set of four arrival times in isolation, ignoring the previous robot position and subsequent motion that may have taken place. The Kalman filter approach also allows the rejection of spurious arrival times which can arise if an indirect path is taken by a pulse from beacon to receiver. Given that the measurement and state noise is Gaussian, the Kalman filter provides the optimum minimum error variance estimate of the position. Moreover, the position estimate is updated each time a beacon fires, thus increasing the frequency of localisation by a factor of four. The estimated error variance of the position estimates is also available from the Kalman filter. Finally, the Kalman filter 
implementation is easily generalised to more that four beacons, for a more robust solution. The cost of these advantages is increased processing time.

On each pulse arrival, the Kalman filter estimates the $n$ dimensional state of the system, denoted $\mathbf{x}$, given a new measurement vector $\mathbf{y}$. The state in our case is the 5 dimensional vector

$$
\mathbf{x}=\left[\begin{array}{lllll}
x & y & z & T & t_{f}
\end{array}\right]^{\mathrm{T}}
$$

which consists of the robot position, $(\mathrm{x}, \mathrm{y}, \mathrm{z})$, the beacon inter-firing period, $T$, and the beacon firing time, $t_{f}$. The state transition equations predict the state at the next time step, $\mathbf{x}(n+1)$, given the current state, $\mathbf{x}(\mathrm{n})$, and allow for uncertainty by incorporating noise components:

$$
\begin{aligned}
\mathbf{x}(n+1) & =\Phi \cdot \mathbf{x}(n)+\mathbf{w}(n+1) \\
& =\left[\begin{array}{lllll}
1 & 0 & 0 & 0 & 0 \\
0 & 1 & 0 & 0 & 0 \\
0 & 0 & 1 & 0 & 0 \\
0 & 0 & 0 & 1 & 0 \\
0 & 0 & 0 & 1 & 1
\end{array}\right] \cdot \mathbf{x}(n)+\left[\begin{array}{c}
w_{x} \\
w_{y} \\
w_{z} \\
w_{T} \\
0
\end{array}\right]
\end{aligned}
$$

Where $\Phi$ is the state transition matrix and $\mathbf{w}(n+1)$ is zero mean white noise with covariance matrix $\mathbf{Q}$. The white noise components $w_{x}, w_{y}$, $w_{z}$ and $w_{T}$ are assumed to be independent with variances $\sigma_{x}{ }^{2}, \sigma_{y}{ }^{2}$, $\sigma_{z}{ }^{2}$ and $\sigma_{T}{ }^{2}$. The standard deviations $\sigma_{x}, \sigma_{y}, \sigma_{z}$ are chosen to be of the same order as the maximum movement anticipated in each axis in one time step, and $\sigma_{T}$ chosen to represent drift in timing due to temperature variations in the beacon control circuitry and local time base of the receiver.

Once state prediction has been performed, the state is then adjusted by the incorporation of a measurement vector. The 
measurement vector is simply the one dimensional arrival time, denoted $t_{i}$ where $i$ is the beacon identity. The formulation of a Kalman filter requires the measurement to be expressed as a function of the state, rather than the more difficult problem of expressing the state in terms of the measurements as has been performed in the previous section. The arrival time $t_{i}$ can be expressed as:

$$
t_{i}=t_{f}+\sqrt{\left(x-x_{i}\right)^{2}+\left(y-y_{i}\right)^{2}+\left(z-z_{i}\right)^{2}} / c
$$

When the function is non-linear, as is the case in equation (11), the Iterated Extended Kalman filter can be employed to iteratively linearise the function about the estimate on each step7,11. The measurement noise variance needs to be specified, and this was measured directly from experiments at fixed locations and found to be approximately constant.

In practice, some measurements are grossly in error due to echoed arrival times. If the direct path between a beacon and the localiser is obscured, and the pulse may take an indirect path, either diffracted around an obstacle or reflected off another object. These echoed arrival times need to be rejected, otherwise divergence of the filter may result or, at best, large errors in position estimation may occur. The criterion for rejection is based on a validation gate 12 which rejects a measurement when the estimated number of standard deviations the measurement is from its expected value (available from the Kalman filter) exceeds a threshold, say 3 standard deviations. A validation gate may cause valid measurements to be rejected occasionally in the normal course of events, and even frequently when the state transition noise variances $\sigma_{x}{ }^{2}, \sigma_{y}{ }^{2}, \sigma_{z}{ }^{2}$ are set unrealistically low for the type of robot motion encountered. 
The geometric approach described in the previous section is unable to reject echoed arrival times when four beacons are employed, since no past data is used as in the Kalman filter nor is there any measurement redundancy in the four arrival times alone. When redundant beacons are employed, beyond the four required, geometric arguments can be used to reject late arrivals as is performed in a $2 \mathrm{D}$ version of the localiser6. The angle of arrival of a pulse, derived from the maximum receiver, can also be used to reject those echoed pulses which arrive from a grossly different direction.

It is possible, but unlikely, that some arrival times are delayed only slightly rather than grossly. This may occur when a diffracted path is taken around a small obstacle that differs little from the direct path to a beacon for example. Unfortunately, there is no way of distinguishing these errors from genuine random noise perturbations of the arrival time unless they persisted for a long period of time. The effect on the Kalman filter is that a small temporary bias will be introduced in the position estimates.

Due to the computation involved with matrix manipulations, the processing time for the Kalman filter algorithm is considerably longer than the previous approach. On a $16 \mathrm{MHz} 80286$, measurements could be processed in real time - that is 230 msec per measurement step. The Kalman filter software was coded in $\mathrm{C}++$ using a class library written by the author applicable to any Kalman filtering application.

Further measurements can easily be incorporated into the localiser Kalman filter, such as dead-reckoning of movements 7 , underwater depth sensors 4 or inertial navigation devices. 


\section{Orientation Determination}

For each beacon firing, the receiver array reports the closest receiver transducer to the beacon. These direction measurements are used for determining the orientation of the robot.

Two approaches, along the lines of the position determination, are considered for orientation determination. The first technique relies on a set of four direction measurements to geometrically evaluate the orientation of the receiver array. The second technique employs an Iterated Extended Kalman filter which allows measurements to be smoothed in accordance with knowledge of motion statistics.

\subsection{Geometrical Orientation Algorithm}

The orientation of the robot can be determined from the arrival directions of pulses with respect to the robot from two beacons and the robot position. The 3-D rotational transformation that aligns the two arrival direction vectors in local robot coordinates with vectors to the two beacons in global coordinates is derived below.

The derivation proceeds in three stages. Firstly, the general form of the transformation that rotates a given vector to another vector about the three dimensional axis perpendicular to both vectors is derived. Stage two uses such a transformation to match the normals of the first to the second of two planes defined by: (i) the two local arrival direction vectors and (ii) the two beacon global vectors. Thirdly, working on the now common plane, the transformation that matches the bisectors of the vectors (ii) and the transformed vectors (i) is derived. The bisector is chosen since the angles between the vectors in (i) and (ii) may not be exactly equal. 
Three dimensional vectors are represented in the form of column vectors $\mathbf{v}=\left[\begin{array}{ll}\mathrm{x} y z & \mathrm{z}\end{array}\right]^{\mathrm{T}}$. The orientation is obtained from the $3 \mathrm{x} 3$ transformation matrix Tfinal, derived below. Each column of Tfinal represents the directions of robot axes in the world coordinate frame.

Firstly, the transformation is derived that rotates a unit vector $\mathbf{v}$ to a unit reference vector vref about the axis orthogonal to both vectors. This is defined by a rotation of angle $\alpha$ about the axis $\mathbf{v} \times \mathbf{v r e f}$, where $\alpha$ is defined by $\sin (\alpha)=\|\mathbf{v} \times \mathbf{v r e f}\|$ and $\cos (\alpha)=\mathbf{v} \bullet \mathbf{v r e f}$. The transformation matrix $\mathbf{T}$ can be obtained by a combination of rotations about the coordinate axes that are described in 13 (in terms of row vectors rather than the column vectors used here):

$$
\mathbf{T}(\mathbf{v}, \mathbf{v r e f})=R_{x}^{-1} \cdot R_{y}^{-1} \cdot R_{\alpha} \cdot R_{y} \cdot R_{x}
$$

where $R_{x}^{-1}=R_{x}^{T}$ and $R_{y}^{-1}=R_{y}{ }^{T}$ are given below in terms of the direction cosines of the axis of rotation $[\mathrm{cx} \mathrm{cy} \mathrm{cz}]=\operatorname{unit}(\mathbf{v} \times \mathbf{v r e f})$ :

$$
\begin{aligned}
& \mathbf{R}_{\mathbf{x}}=\left[\begin{array}{ccc}
1 & 0 & 0 \\
0 & \frac{c z}{\sqrt{c x^{2}+c y^{2}}} & \frac{-c y}{\sqrt{c x^{2}+c y^{2}}} \\
0 & \frac{c y}{\sqrt{c x^{2}+c y^{2}}} & \frac{c z}{\sqrt{c x^{2}+c y^{2}}}
\end{array}\right] \\
& \mathbf{R}_{\mathbf{y}}=\left[\begin{array}{ccc}
\sqrt{c x^{2}+c y^{2}} & 0 & -c x \\
0 & 1 & 0 \\
c x & 0 & \sqrt{c x^{2}+c y^{2}}
\end{array}\right] \\
& \mathbf{R}_{\alpha}=\left[\begin{array}{ccc}
\cos (\alpha) & -\sin (\alpha) & 0 \\
\sin (\alpha) & \cos (\alpha) & 0 \\
0 & 0 & 1
\end{array}\right]
\end{aligned}
$$

The rotation transformation to match normal vectors of planes defined by the receiving elements $\mathbf{n} \mathbf{1}$ and $\mathbf{n} \mathbf{2}$ with the unit vectors to 
the corresponding beacons $\mathbf{b} \mathbf{1}$ and $\mathbf{b} \mathbf{2}$ is derived below. If either $\mathbf{n} \mathbf{1}$ and $\mathbf{n} \mathbf{2}$ or b1 and $\mathbf{b 2}$ lie in a straight line, there is no unique rotation to match the vector pairs and the problem is ill-conditioned. Thus the pairs of vectors are chosen from a cycle of four beacons to have the maximum absolute sine of angle between them. The planes defined by the vector pairs are matched with Tplanes given by

$$
\text { Tplanes }=\mathbf{T}(\text { unit }(\mathbf{n} 1 \times \mathbf{n} 2), \operatorname{unit}(\mathbf{b} 1 \times \mathbf{b 2}))
$$

The angles between the pairs may not be the same in practice due to the discretisation in the receiver normal vectors (ie there are only 32 of them for a full solid angle). The approach taken is to match the pairs so that the error is minimum and the same for each vector. The bisector vectors, which now lie in the same plane, are matched, to produce the final transformation:

$$
\text { Tfinal }=\mathbf{T}(\mathbf{T} \text { planes. } \operatorname{unit}(\mathbf{n} 1+\mathbf{n} 2), \operatorname{unit}(\mathbf{b} 1+\mathbf{b} 2)) . \text { Tplanes }
$$

The transformation Tfinal can be converted to the roll, pitch and yaw angles (described in the next section) by referring to Graig 14 .

\subsection{Iterated Extended Kalman Filter for Orientation Estimation}

There are three degrees of freedom of orientation, and these are represented by nine elements of the transformation matrix described in the previous section. Such a redundant state representation in a Kalman filter implementation would result in complexity and inefficiency. Instead, the orientation is represented by a state vector consisting of roll, pitch and yaw angles, $\phi, \theta, \psi 15$. The transformation matrix $\operatorname{RPY}(\phi, \theta, \psi)$ that rotates the world axis frame to the axes of the 
robot is defined by the ordered rotations around each coordinate axis as follows:

$\operatorname{RPY}(\phi, \theta, \psi)=\operatorname{Rot}(z, \phi) \operatorname{Rot}(y, \theta) \operatorname{Rot}(x, \psi)$

where $\operatorname{Rot}(\mathrm{x}, \psi)$ is a rotation around the $\mathrm{x}$-axis by angle $\psi$ and similarly for $\operatorname{Rot}(y, \theta)$ and $\operatorname{Rot}(z, \phi)$. These are defined in terms of matrix transformations in McKerrow ${ }^{15}$. Note that the orientation representation is not unique for roll, pitch and yaw angles between $-\pi$ and $\pi$ and the restriction of a pitch angle lying between $-\pi / 2$ and $\pi / 2$ is necessary to make the representation unique. Furthermore, if the pitch angle is equal to $\pi / 2$ or $-\pi / 2$, only the sum or difference of roll and yaw angles is unique, and then the roll angle can be arbitrarily defined to be zero to achieve a unique representation 14 .

In the Kalman filter implementation, the state transition matrix, $\Phi$, is the identity matrix. The measurement equation relates the direction cosines of the arrival pulse direction relative to the local robot axis frame, to the filter state (roll, pitch and yaw angles). The measurement vector of arrival direction cosines $[\alpha \beta \gamma]^{\mathrm{T}}$ is expressed in terms of the state for beacon $i$ :

$$
\left[\begin{array}{l}
\alpha \\
\beta \\
\gamma
\end{array}\right]=R P Y^{T}(\phi, \theta, \psi) \frac{1}{d_{i}}\left[\begin{array}{l}
x_{i}-x \\
y_{i}-y \\
z_{i}-z
\end{array}\right]
$$

where $R P Y^{T}$ is also the inverse of $R P Y$. The position of the robot $(\mathrm{x}, \mathrm{y}, \mathrm{z})$ is available from the position determination algorithms described above. The noise variances associated with the measurements can be estimated based on the discretisation caused by the 32 receiver 
transducers in the receiver array geometry. It is worth noting that the measurement errors are not strictly independent from sample to sample, since they are dominated by discrepancies between receiver transducer orientation and the actual arrival direction due to receiver angle discretisation. If the robot is stationary these errors will be nearly identical from measurement to measurement. The Kalman filter will then optimistically estimate the state covariance matrix. This covariance matrix approaches the zero matrix if the state transition noise covariances are zero, as is the case when the robot is known to be stationary. If the state error covariance matrix is unrealistically small, future measurements will be underweighted or rejected if a validation gate is in place. A practical solution is to ensure that the state transition covariance matrix is sufficiently large in all cases. The result is a sub-optimal filter that nevertheless performs well in practice. The orientation estimate can be fused with other sensors such as compasses, gravitational field sensors, and inertial navigation devices very simply by using the same Kalman filter structure with additional measurement equations.

\section{Performance of the Prototype}

\subsection{Position Accuracy}

Beacons were positioned at $2600 \mathrm{~mm}$ along each of the coordinate axes in a corner of the laboratory as in figure 1 . The speed of sound was estimated to be $340 \mathrm{~m} / \mathrm{s}$ based on the temperature readings. The $3 \mathrm{D}$ position was measured manually with a tape measure at five positions and results of the localiser collected and summarised in Table I and Table II. The apparent errors are due to

- tape measurement errors of up to $10 \mathrm{~mm}$. 
- arrival time estimation errors of the ultrasonic pulses due to the simple envelope thresholding technique and the slow rise time of approximately $500 \mu \mathrm{sec}$ of the piezzo-electric transducers employed in the prototype. These errors can be improved by using computational expensive correlation techniques outlined in references 16,17 .

- error in estimating the speed of sound, which can be as much as 1 percent when temperature compensation is used alone ${ }^{18}$.

- air turbulence and local variations in the speed of sound.

The Kalman filter results in Table II assumed that the robot was stationary with arrival time standard deviation of $0.2 \mathrm{msec}$ and a validation gate of 2 standard deviations. Note that the Kalman filter results are usually closer to the measured position than the geometric approach, especially in the last row of the tables, where the validation gate of the Kalman filter rejected 50 spurious echoed arrival times. 
TABLE I - Localiser Results using Geometric Position Algorithm

\begin{tabular}{|c|c|c|c|c|c|c|}
\hline \multirow{2}{*}{$\begin{array}{l}\text { Numbe } \\
\text { r } \\
\text { Sample } \\
\text { s }\end{array}$} & \multicolumn{3}{|c|}{$\begin{array}{l}\text { Mean Localiser Reported Position } \\
\text { (standard deviation of results) } \mathrm{mm}\end{array}$} & \multicolumn{3}{|c|}{$\begin{array}{c}\text { Measured } \\
\text { Position mm } \\
\end{array}$} \\
\hline & $\mathrm{x}$ & $\mathrm{y}$ & $z$ & $\mathrm{x}$ & $\mathrm{y}$ & $z$ \\
\hline 111 & $1207(7.3)$ & $1116(10.0)$ & $1346(7.1)$ & $\begin{array}{l}121 \\
0\end{array}$ & $\begin{array}{l}115 \\
0\end{array}$ & $\begin{array}{l}136 \\
0\end{array}$ \\
\hline 68 & $1186(7.7)$ & $1121(6.9)$ & $\begin{array}{r}916 \\
\\
(6.35) \\
\end{array}$ & $\begin{array}{l}121 \\
0\end{array}$ & $\begin{array}{l}113 \\
0\end{array}$ & 900 \\
\hline 65 & 567 (8.8) & $1124(5.4)$ & $901(8.6)$ & 590 & $\begin{array}{l}114 \\
0\end{array}$ & 900 \\
\hline 64 & $\begin{array}{l}2335 \\
(78.1)\end{array}$ & 2077 (67.6) & $\begin{array}{l}1341 \\
(15.7)\end{array}$ & $\begin{array}{l}237 \\
0\end{array}$ & $\begin{array}{l}220 \\
0\end{array}$ & $\begin{array}{l}135 \\
0\end{array}$ \\
\hline 80 & $586(9.7)$ & $1006(122.0)$ & $\begin{array}{l}1577 \\
(10.6) \\
\end{array}$ & 600 & $\begin{array}{l}115 \\
0 \\
\end{array}$ & $\begin{array}{l}160 \\
0 \\
\end{array}$ \\
\hline
\end{tabular}

TABLE II - Localiser Results using Kalman Filter

\begin{tabular}{||l|l|l|l|l|l|l||}
\hline $\begin{array}{l}\text { Numbe } \\
\text { r } \\
\text { Sample } \\
\mathrm{s}\end{array}$ & $\begin{array}{r}\text { Localiser Reported Position (standard } \\
\text { deviation from filter) } \mathrm{mm}\end{array}$ & \multicolumn{3}{|c||}{ Measured } \\
\cline { 2 - 8 } & $\mathrm{x}$ & $\mathrm{y}$ & $\mathrm{z}$ & $\mathrm{x}$ & $\mathrm{y}$ & $\mathrm{z}$ \\
\hline 111 & $1210(7.4)$ & $1122(7.5)$ & $1357(7.1)$ & $\begin{array}{l}121 \\
0\end{array}$ & $\begin{array}{l}115 \\
0\end{array}$ & $\begin{array}{l}136 \\
0\end{array}$ \\
\hline 68 & $1188(8.3)$ & $1127(8.5)$ & $926(9.0)$ & $\begin{array}{l}121 \\
0\end{array}$ & $\begin{array}{l}113 \\
0\end{array}$ & 900 \\
\hline 65 & $569(9.6)$ & $1130(7.4)$ & $911(8.1)$ & & $\begin{array}{l}114 \\
590\end{array}$ & \\
\hline
\end{tabular}




\begin{tabular}{||l|l|l|l|l|l|l||}
\hline 64 & $\begin{array}{l}2341 \\
(21.8)\end{array}$ & $2089(19.8)$ & $\begin{array}{l}1358 \\
(15.1)\end{array}$ & $\begin{array}{l}237 \\
0\end{array}$ & $\begin{array}{l}220 \\
0\end{array}$ & $\begin{array}{l}135 \\
0\end{array}$ \\
\hline 80 & $574(9.7)$ & $1109(10.1)$ & $1593(7.3)$ & $\begin{array}{l}115 \\
160 \\
0\end{array}$ \\
\hline
\end{tabular}

\subsection{Orientation Accuracy}

The orientation estimate is obtained from the closest receiver to each beacon. The dominant errors in this measurement process are due to the inherent discrete sampling of angle, rather than by random processes. These errors are deterministic since they can be determined a priori from the orientation and position. An upper bound on the angle discretisation error is 21 degrees which is half the maximum angular separation of the receivers, assuming that the receiver channels are matched. An unlikely situation can occur where all beacons are seen exactly halfway between pairs of receivers and the geometric orientation algorithm could report angles in error by 21 degrees. The Kalman filter orientation error may also produce this error if no movement occurs. Localiser movement results in the Kalman filter algorithm averaging data with time and the reduction of errors.

The orientation errors due to the discretisation of received angle are studied by a Monte-Carlo analysis. A random walk in position and angle is generated with a standard deviation of $5 \mathrm{~mm}$ on each axis and 0.57 degrees in roll, pitch and yaw for each beacon firing. The position was restricted to lie in a cube bounded by the points $(0.5$, $0.5,0.5)$ and $(2.5,2.5,2.5)$ meters. The angle error on each measurement is defined as the maximum angle difference between the axes of the actual localiser coordinate frame and the measured localiser coordinate frame. The results are summarised in Table III for 
10000 samples each of the Extended Kalman Filter (EKF) angle estimate and the geometrical angle estimate. Note that the EKF performs significantly better than the geometrical technique since all four beacon directions are used, compared to the two out of four in the geometrical technique. Also the EKF incorporates past information into its estimate of orientation since the orientation changes slowly. The geometrical approach estimates orientation after collecting a full cycle of 4 beacon measurements under the assumption that the localiser is stationary. However, in practice and indeed in the Monte-Carlo analysis the orientation changes after each transmitter firing. This explains why the maximum error is larger than that of the EKF. The maximum EKF angle error is due to the discretisation of angle with the receiver spacing of 42 degrees as described above.

TABLE III - Orientation Errors.

\begin{tabular}{|c|c|c|}
\hline & $\begin{array}{l}\text { Maximum Angle } \\
\text { Error (degrees) }\end{array}$ & $\begin{array}{c}\text { Mean Angle Error } \\
\text { (degrees) }\end{array}$ \\
\hline EKF angle estimate & 21.1 & 9.34 \\
\hline $\begin{array}{l}\text { Geometrical angle } \\
\text { estimate }\end{array}$ & 29.7 & 15.0 \\
\hline
\end{tabular}

\section{Future Work and Conclusions}

A novel three dimensional localiser has been designed, constructed and tested. The laboratory prototype performs to an accuracy of approximately $50 \mathrm{~mm}$ depending on the position. The size of the prototype workspace could be extended to 10 meters on each axis with no modification of the hardware (if laboratory space permitted) and with little change in the absolute error, based on 
experience with a two dimensional ultrasonic localiser ${ }^{7}$. Improvements in the accuracy of the localiser can be achieved by more sophisticated pulse arrival time detection techniques 16,17 and the use of more expensive transducers. However the aim of this prototype was to prove the concept and this has been successfully achieved.

Further beacons can also be added beyond the minimum required number of four to improve the robustness and accuracy of the localiser. Kalman filtering techniques are particularly suited to fusing the data of redundant beacons and also the exclusion of spurious arrival times. An underwater version is also being considered as an extension of this work.

\section{Acknowledgments}

The helpful suggestions of Dr Greg Cambrell in the derivation of the iterative position determination algorithm are gratefully acknowledged. The construction of the prototype was assisted by Shaun Geddes.

\section{References}

1. $\mathrm{R}$ V Ifremer-irisa and M L Lib-ubo, "Absolute location of underwater robot vehicles by acoustic data fusion", IEEE International Conference on Robotics and Automation, Cincinnati, Ohio pp 1310-1315, May 13-18 1990.

2. D R Yoerger and $\mathrm{J} E$ Slotine, "Adaptive sliding control of an experimental underwater vehicle", IEEE International Conference on Robotics and Automation, Sacramento, California pp 2746-2751, April 1991. 
3. $\mathrm{J} J$ Leonard and $\mathrm{J}$ G Bellimgham, "Directed sensing strategies for feature-relative navigation", Sensor Fusion VI (SPIE Volume 2059), Boston MA, pp. 120-129, Sept 71993.

4. J G Bellingham, T R Consi, U Tedrow and Diane Di Massa, "Hyperbolic acoustic navigation for underwater vehicles: implementation and demonstration", IEEE AUV'92, Washington DC, pp 304-309, June 1992.

5. J Hallam, P Forster and $\mathrm{J}$ Howe, "Map-free localisation in a partially moving 3D world: the Edinburgh feature-based navigator", Proceedings of IAS-2, Amsterdam, pp. 726-736, 1988.

6. L Kleeman, "Ultrasonic autonomous robot localisation system", IEEE international conference Intelligent Robots and Systems '89 Tsukuba, JAPAN, pp.212-219 September 1989.

7. L.Kleeman, "Optimal estimation of position and heading for mobile robots using ultrasonic beacons and dead-reckoning", IEEE International Conference on Robotics and Automation, Nice, France, pp 2582-2587, May 10-15 1992.

8. L Kleeman, "Iterative algorithm for three dimensional autonomous robot localisation", Third National Conference on Robotics, Melbourne pp. 210-219 June 1990.

9. L.Kleeman, "A three dimensional localiser for autonomous robot vehicles", IEEE Region 10 Conference, Melbourne November 1992, pp 860-864.

10. R L Frank, "Current developments in Loran-C", Proceedings of the IEEE, Vol 71, No 10, October 1983, pp. 1127-1139.

11. A. H. Jazwinski, Stochastic Processes and Filtering Theory, (Mathematics in Science and Engineering, Vol. 64), Academic Press, New York, 1970. 
12. J. F. Leonard, H. F. Durrant-Whyte, "Mobile robot localization by tracking geometric beacons", IEEE Transactions Robotics and Automation Vol. 7 No. 3, pp 376-382, June 1991.

13. D F Rogers and J A Adams, Mathematical Elements for Computer Graphics 2nd Edn, McGraw-Hill, New York, 1990.

$14 \mathrm{~J} \mathrm{~J}$ Graig, Introduction to Robotics, Mechanics and Control, Addison-Wesley, Reading, 1989.

15. P J McKerrow Introduction to Robotics, Addison-Wesley, Sydney, 1991.

16. M Parilla, J J Anaya and C Fritsch, "Digital signal processing techniques for high accuracy ultrasonic range measurements", IEEE Trans on Instrumentation and Measurement, Vol 40, no 4 Aug 1991, pp. 759-763.

17. D Marioli, C Narduzzi, C Offelli, D Petri, E Sardini and A Taroni, "Digital time-of-flight measurement for ultrasonic sensors", IEEE Trans on Instrumentation and Measurement, Vol 41, no 1, Feb 1992, pp. 93-97.

18. H H Poole, Fundamentals of Robotics Engineering, Van Nostrand Reinhold, New York 1989. 